\title{
Clinical and histological evolution after de novo donor-specific anti-human leukocyte antigen antibodies: a single centre retrospective study
}

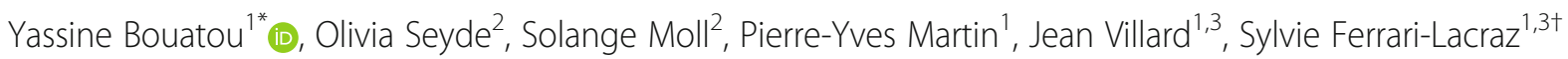
and Karine Hadaya ${ }^{1,4+}$

\begin{abstract}
Background: Donor-specific anti-human leukocyte antigen (HLA) antibodies (DSA) can be preformed or de novo (dn). Strategies to manage preformed DSA are well described, but data on the management and outcomes of dnDSA are lacking.

Methods: We performed a retrospective analysis of data from a single centre of the management and outcomes of 22 patients in whom a dnDSA was identified with contemporary and follow up biopsies.

Results: Evolution from baseline to follow up revealed a statistically significant loss of kidney function (estimated glomerular filtration rate: $45.9 \pm 16.7$ versus $\left.37.4 \pm 13.8 \mathrm{ml} / \mathrm{min} / 1.73 \mathrm{~m}^{2} ; p=0.005\right)$ and increase in the proportion of patients with transplant glomerulopathy (percentage with cg lesion $\geq 1: 27.2 \%$ vs. $45.4 \% ; p=0.04$ ). Nine patients were not treated at the time of $\operatorname{nnDSA}$ identification, and 13 patients received various drug combinations (e.g., corticosteroids, plasmapheresis, thymoglobulins and/or rituximab). No significant pathological changes were observed for the various treatment combinations.

Conclusion: Our retrospective analysis of a small sample suggests that dnDSA should be considered a risk factor for the loss of kidney function independent of the baseline biopsy, and multidisciplinary evaluations of the transplant patient are a necessary requirement. Further confirmation in a multicentre prospective trial is required.
\end{abstract}

Keywords: de novo DSA, Renal pathology, HLA-antibody post-transplantation, Outcome

\section{Background}

Kidney transplantation (KT) remains associated with suboptimal 5- and 10-year graft survival (77\% and 56\%, respectively [1]), despite an excellent 1-year allograft survival (91\%). Graft failure is primarily associated with antibody mediated rejection (ABMR) [2-4].

Donor-specific anti-human leukocyte antigen (HLA) antibodies (DSA) drive ABMR. Development of the solid phase assay (SPA) to detect DSA was significant in the

\footnotetext{
* Correspondence: yrbouatou@gmail.com

${ }^{\dagger}$ Equal contributors

${ }^{1}$ Division of Nephrology, Geneva University Hospitals, Rue Gabrielle

Perret-Gentil 4, 1205 Geneva, Switzerland

Full list of author information is available at the end of the article
}

diagnosis of DSA [5]. SPA can detect DSA at the time of transplantation even with a negative flow cytometry crossmatch. DSA is divided into two categories: preformed DSA and de novo DSA ( $d n$ DSA). Preformed DSA may be associated with hyperacute rejection or ABMR in the weeks following transplantation. The presence of preformed DSA is associated with graft failure [6-8].

DSA development after KT is known as $d n$ DSA. A previous review [9] noted that $d n \mathrm{DSA}$ primarily occurred during immunosuppression [10] in the context of poor adherence $[4,11,12]$. Early calcineurin inhibitor minimization may also be associated with $d n$ DSA development [9]. Wiebe et al. [12] reported a trend towards an association with a history of acute rejection as an

(c) The Author(s). 2018 Open Access This article is distributed under the terms of the Creative Commons Attribution 4.0 International License (http://creativecommons.org/licenses/by/4.0/), which permits unrestricted use, distribution, and 
independent risk factor for $d n \mathrm{DSA}$. DnDSA is independently associated with poor long-term allograft outcomes [13]. SPA technique allows to further detect complement-binding DSA, and these DSAs are associated with reduced graft survival compared to noncomplement-binding DSA [14, 15].

Knowledge of the presence of DSA at the time of transplantation allows implementation of specific therapeutic strategies (e.g., desensitization) to reduce the levels of preformed DSA [16]. Several studies demonstrated acceptable long-term patient survival compared to wait-listed patients on dialysis [16, 17]. However, the therapeutic management of $d n \mathrm{DSA}$ remains controversial. Plasma exchange, intravenous immunoglobulin (IVIG), anti-thymoglobulin antibodies (ATG), rituximab, bortezomib and treatment abstention are used in different combinations for the treatment of ABMR [18]. Data on the long-term efficacy of these regimens and treatment strategies guided by the presence of $d n \mathrm{DSA}$ (independent histology) are lacking.

The present study retrospectively analysed the diagnosis and clinical and histological evolution in patients who developed $d n$ DSA.

\section{Methods}

\section{Patient selection and follow up}

We retrospectively analysed the stored sera available from all consecutive patients who underwent kidney transplantation in Geneva University Hospitals from 1986 to 2012. Patients with combined transplantation (kidney - liver, kidney - heart or kidney - pancreas) were excluded. The cumulative incidence of $d n \mathrm{DSA}$ is $15 \%$ in our centre. We identified 22 patients with known $d n$ DSA from the 315 patients transplanted from 1986 to 2012 for whom we had available sera. DnDSA was defined as a DSA that developed within six months after kidney transplantation with a mean fluorescence intensity $(\mathrm{MFI})>500$. All 22 patients were transplanted with a negative $\mathrm{CDC}$ crossmatch ( $\mathrm{B}$ and $\mathrm{T}$ ) and/or FACS crossmatch. Clinical data were recorded during regular follow up at our institution. Graft function was assessed using the estimated glomerular filtration rate (eGFR). We used the CKD-EPI formula for each patient. The eGFR was calculated at the time of biopsy. A patient's return to haemodialysis defined graft failure. We analysed graft biopsies at the time of $d n$ DSA detection ( \pm 3 months) in these patients and therapy administration according to the histological data. We identified a positive DSA in 2012 and retrospectively evaluated the sera from these patients to identify the first positive serum and matched the biopsy at the time of first identification of the $d n \mathrm{DSA}$. The biopsies matched with the $d n \mathrm{DSA}$ were a protocol or indication biopsy because of the retrospective nature of this study.
The ethical committee approved the study ( $\left.\mathrm{N}^{\circ} 6-208\right)$ which is in accordance with the regulations of the Geneva University Hospitals.

Immunosuppression dnDSA/histological-based treatment Induction therapy evolved over time and consisted of no induction or basiliximab on post-operative days (PODs) 0 and 4. Antithymocyte globulin (ATG) or daclizumab reflected the use of a steroid-free protocol in selected patients. The immunosuppressive regimen consisted of tacrolimus or cyclosporine A, corticosteroids, and mycophenolate mofetil, mycophenolic acid or azathioprine. We reported the identified treatment combination at the time of $d n$ DSA and biopsy as reported in medical files. The reported combinations included high doses of steroids, ATG, anti-CD20 and/or plasma exchange. These treatments were adjusted according to histopathological findings and patient's age and comorbidities (e.g., cancer, previous infections).

\section{Graft biopsies}

Protocol biopsies are performed in our centre at one year post-transplantation, but the present analysis focused on kidney biopsies performed at approximately the time of development of $d n \mathrm{DSA}$, regardless of kidney function. The clinician may not have known of the presence of $d n$ DSA at the time of the biopsy. A control biopsy at the last follow up was also included to evaluate the histological changes following treatment of the $d n$ DSA. The next available graft biopsy was used in patients who were not treated. Criteria from the Banff 2013 classification were used for the retrospective grading of biopsies [19].

\section{Determination of DSA using Luminex solid-phase assay}

Patient sera were analysed for the presence of anti-HLA class I and class II antibodies using solid phase assays on Luminex and the Labscreen Mix assay for HLA class I and HLA class II following the recommendations of the manufacturer (One Lambda, Canoga Park, CA), as previously described [20]. Sera collected before and after transplantation of all positive individuals were subsequently tested for anti-HLA class I- and class II-specific antibodies using the Luminex single antigen beads (one Lambda). Briefly, colour-coded microspheres coated with major HLA class I and II antigens were incubated with $10 \mu \mathrm{l}$ serum for $30 \mathrm{~min}$ at room temperature in the dark. Samples were washed three times and incubated with $100 \mu \mathrm{L}$ of $1: 100$ phycoerythrin-conjugated goat anti-human IgG (One Lambda Inc.). Samples were washed twice, and the fluorescent signal intensity for each microsphere was measured using a LABScan 100 flow analyser (One Lambda Inc.). The cut-off for positive samples was the normalized background (NBG) ratio 
recommended by the manufacturer, which was performed using HLA Fusion software (One Lambda). An MFI $>500$ was considered positive, as recommended by the manufacturer, and clinical relevance was considered at MFI $>1000$. Donor-specific antigens were classified as immunogenic HLA or non-immunogenic HLA based on the presence of specific antibodies in the recipient directed towards the donor-specific antigens.

Post-transplantation screening consisted of Luminex solid-phase assays performed at 1 month, 3 months, 6 months, 9 months, 12 months and annually. Each increase in serum creatinine $>25 \%$ required a new Luminex solid-phase assay.

\section{Statistical analysis}

Statistical analyses were performed using STATA v. 14.0 (College Station, TX). We used descriptive statistics to estimate the frequencies (\%) and means $( \pm \mathrm{SD})$ of study variables. Comparisons of two means were performed using $t$ tests, and comparisons of the frequencies between groups were evaluated using Fisher's exact test because of the small sample size. We did not perform further analyses because of the retrospective nature of the descriptive data.

\section{Results}

\section{Patients' characteristics}

Table 1 summarises the patients' characteristics. The characteristics of these patients were similar to the transplanted patients who do not develop $d n$ DSA (data not shown). The mean age was 45 years (standard deviation (SD): \pm 14 ), and males predominated (14/22; 63 . $6 \%)$. These kidney transplantations were primarily first ABO compatible, with greater than 3 HLA mismatches. A delayed graft function was observed in $8 / 22$ patients (36.3\%), and $8 / 22$ patients (36.3\%) did not receive induction therapy. Most patients who developed $d n$ DSA received a triple immunosuppression regimen that was prescribed at baseline (21/22 patients; 95.4\%). Eight of the 22 patients $(36.3 \%)$ were treated for a previous episode of T-cell-mediated rejection (TCMR).

\section{Characteristics of the dnDSA}

Table 2 describes the characteristics of the $d n$ DSA. DSA was primarily directed against MHC class II antigens in $15 / 22$ patients $(68.1 \%)$ or in association with Class I $d n$ DSA $(6 / 22 ; 27 \%)$ with a mean MFI of 5896 . No difference in the mean MFI for $d n D S A$ was observed from baseline to follow up (Fig. 1). Notably, 18 patients (81.8\%) received a triple immunosuppressant regimen one year post-kidney transplantation, but only 5 of the 22 patients (22.7\%) received the triple therapy at the time of $d n$ DSA detection. Less than $20 \%$ of the 22 patients with $d n$ DSA exhibited concomitant acute kidney injury at the time
Table 1 Characteristics of patients and transplantations with late de novo DSA

\begin{tabular}{|c|c|}
\hline & $\begin{array}{l}\text { Patients with late } d n D S A \\
(n=22)\end{array}$ \\
\hline \multicolumn{2}{|l|}{ Recipients' characteristics } \\
\hline Age in years; mean (SD) & $45(14)$ \\
\hline Gender (\% male) & 63.6 \\
\hline First transplantation (\%) & 100 \\
\hline Living donor (\% yes) & 36.3 \\
\hline \multicolumn{2}{|l|}{ Baseline nephropathy (\%) } \\
\hline$\circ$ ADPKD & 31.5 \\
\hline $\begin{array}{l}\text { o Glomerulopathy other than IgA } \\
\text { nephropathy }\end{array}$ & 18.3 \\
\hline ○ IgA nephropathy & 18.3 \\
\hline - Diabetes mellitus and/or hypertension & 13.6 \\
\hline o Others & 18.3 \\
\hline \multicolumn{2}{|l|}{ Transplantation characteristics } \\
\hline $\begin{array}{l}\text { HLA mismatches (\% patients with } \\
>\text { or }=3 \text { mismatches) }\end{array}$ & 100 \\
\hline ABO incompatible (n) & 1 \\
\hline Delayed graft function (n, \%) & $8(36.3)$ \\
\hline \multicolumn{2}{|l|}{ Induction therapy $(\mathrm{n}, \%)$} \\
\hline$\circ$ No induction & $8(36.3)$ \\
\hline - Basiliximab & $8(36.3)$ \\
\hline$\circ$ ATG & $5(22.7)$ \\
\hline - Daclizumab & $1(4.5)$ \\
\hline $\begin{array}{l}\text { Initial therapy including a triple regimen }{ }^{a} \\
(\mathrm{n}, \%)\end{array}$ & $21(95.4)$ \\
\hline $\begin{array}{l}\text { Acute rejection or TCMR prior to } \\
\text { development of } d n D S A(n, \%)\end{array}$ & $8(36.3)$ \\
\hline
\end{tabular}

of $d n \mathrm{DSA}$, which was defined as an increase in creatinine $\geq 25 \%$ from baseline and/or appearance of proteinuria $>0.5 \mathrm{~g} / 24 \mathrm{~h}$ at the time of $d n \mathrm{DSA}$ with a mean eGFR of $45.9 \mathrm{ml} / \mathrm{min} / 1.73 \mathrm{~m}^{2}$ (SD: \pm 16.7 ). Half of the patients exhibited albuminuria at the discovery of $d n$ DSA.

\section{Histological parameters before and after therapy}

A median time of 55.5 days was observed between first $d n$ DSA identification and the initial graft biopsy. The range was 102 days prior to the biopsy to 863 days after the biopsy. Figure 2 shows the diagnosis at the initial and the follow up biopsies. We observed that 12/22 (54. $5 \%$ ) patients had a diagnosis of acute ABMR, chronic ABMR or mixed rejection at the time of $d n$ DSA.

Follow up biopsies occurred at a median of 812 days after the baseline biopsy (392; 1624). Most patients 
Table 2 Characteristics of late de novo DSA (dnDSA)

\begin{tabular}{|c|c|}
\hline & $\begin{array}{l}\text { Patients with late } d n D S A \\
(n=22)\end{array}$ \\
\hline $\begin{array}{l}\text { Time from kidney transplantation to } \\
\text { dnDSA in years; mean (SD) }\end{array}$ & $10(7.5)$ \\
\hline \multicolumn{2}{|l|}{ HLA class (n, \%) } \\
\hline Class I & $1(4.5)$ \\
\hline Class II & $15(68.1)$ \\
\hline Class I+ II & $6(27.0)$ \\
\hline MFI mean (SD) & $5896(3879)$ \\
\hline $\begin{array}{l}\text { Triple immunosuppression } 1 \text { year } \\
\text { post-Tx }(n, \%)\end{array}$ & $18(81.8)$ \\
\hline $\begin{array}{l}\text { Triple immunosuppression at the } \\
\text { time of } d n D S A(\%)\end{array}$ & $5(22.7)$ \\
\hline AKI at the time of $\operatorname{dnDSA}(n, \%)$ & $4(18.2)$ \\
\hline $\begin{array}{l}\text { Mean eGFR using the CKD-EPI } \\
\text { equation in } \mathrm{ml} / \mathrm{min}(+/-\mathrm{SD})\end{array}$ & $45.9(16.7)$ \\
\hline $\begin{array}{l}\text { Albuminuria at the time of dnDSA } \\
\text { diagnosis }(n, \%)\end{array}$ & $12(54.5)$ \\
\hline
\end{tabular}

MFI mean fluorescence intensity, $T x$ transplantation, $A K I$ acute kidney injury, eGFR estimated glomerular filtration rate, CKD-EPI Chronic Kidney Disease Epidemiology Collaboration, $S D$ standard deviation presented with chronic active ABMR and transplant glomerulopathy at the follow up biopsy $(11 / 22 ; 50 \%)$.

We did not observe a significant change in microvascular inflammation (MVI) parameters (g, ptc separately, $\mathrm{g}+\mathrm{ptc}$ ) or $\mathrm{C} 4 \mathrm{~d}$ on two consecutive biopsies (Table 3). However, a significant increase in the proportion of patients with a chronic transplant glomerulopathy (cg) score $\geq 1$ from $27.2 \%$ to $45.4 \%(p=0.04)$ was observed between the two biopsies (Table 3).

The eGFR dropped from a mean of 45.9 (SD: \pm 16.7$)$ at the first biopsy to $37.4 \mathrm{ml} / \mathrm{min} / 1.73 \mathrm{~m}^{2}$ (SD: \pm 13.8$)$ at the follow up biopsy (Table $3 ; p=0.005$ ).

Thirteen of the 22 patients $(59.1 \%)$ received a therapeutic regimen that included a steroid pulse $(N=8)$, plasma exchange $(N=6)$, IVIG $(N=5)$ and/or rituximab $(N=6)$. Evaluation of the overall effect of any treatment versus no treatment at the time of $d n \mathrm{DSA}$ on the evolution of MVI and cg score in the follow-up biopsy (Table 4) revealed no statistically significant difference. Treatment did not significantly influence the evolution of eGFR (Table 4).

We analysed each specific drug and the evolution of MVI score (Table 5) or cg score (Table 6). Steroids were associated with a reduction in the MVI score

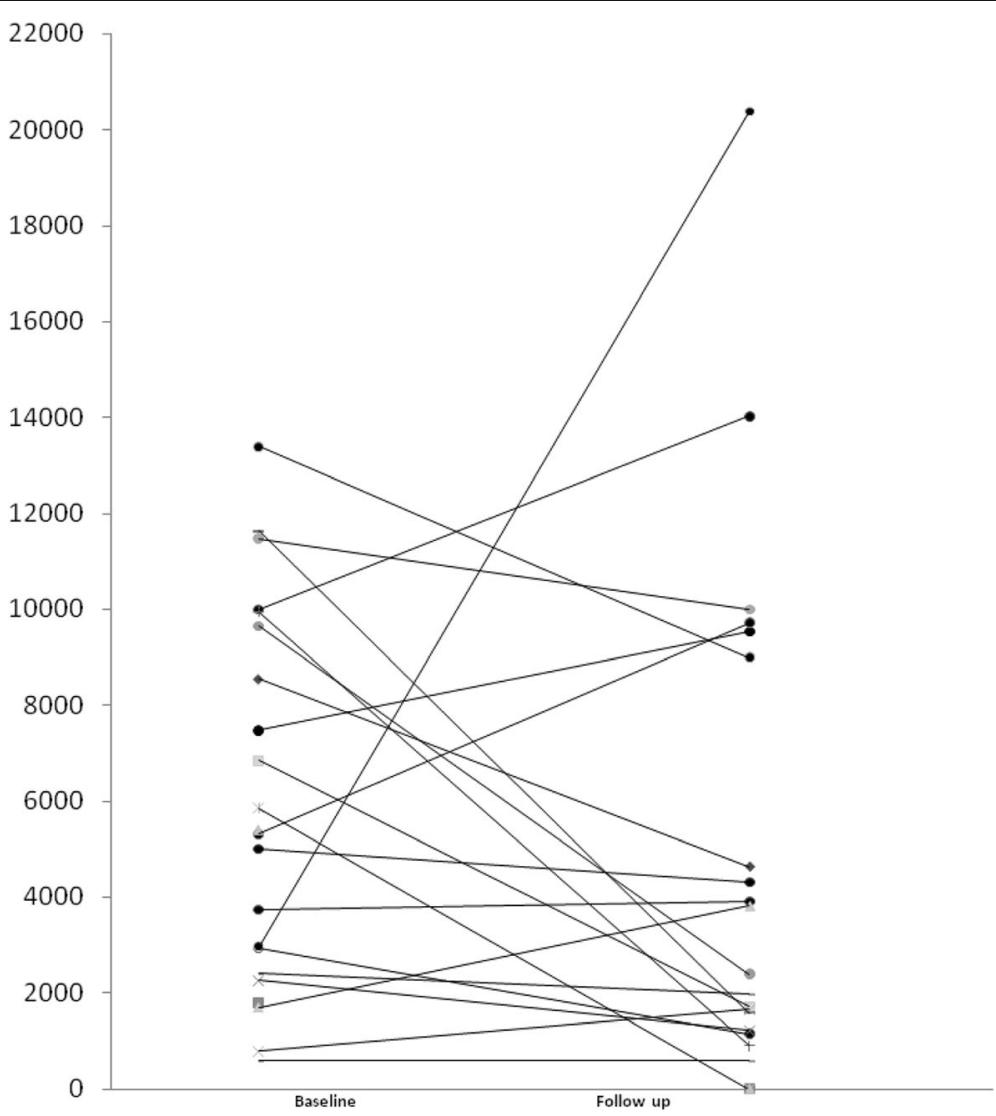

Fig. 1 Individual evolution of dnDSA values in mean fluorescence intensity (MFI) 


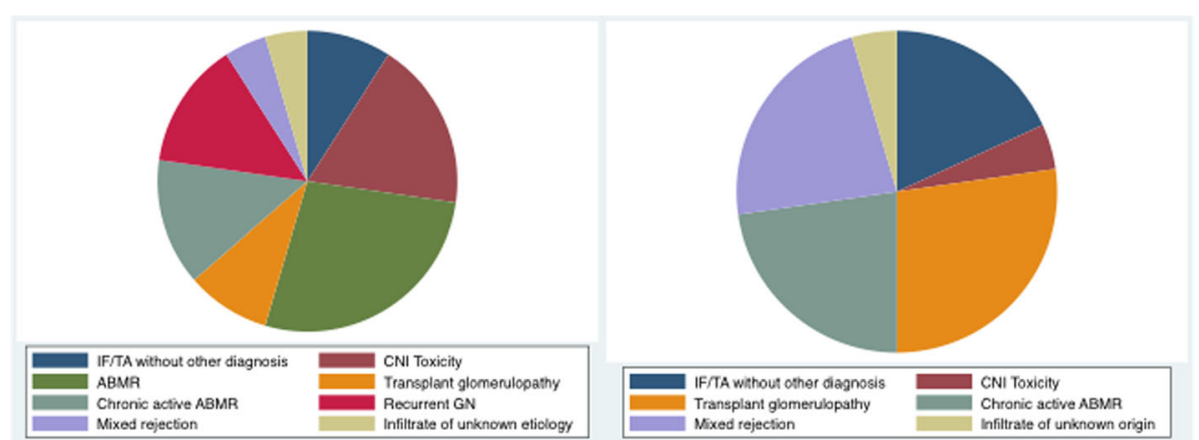

Fig. 2 Final reported diagnosis at baseline and follow up biopsy. IF/TA: interstitial fibrosis with tubular atrophy; CNI: calcineurin inhibitor; ABMR: antibody mediated rejection; GN: glomerulonephritis

(Trend only, $p>0.05$ ), and IVIG therapy was associated with a progression of MVI score $(p<0.05)$. None of the other treatments were significantly associated with a modification of scores even considering a clinically relevant baseline score for MVI (Table 5) and cg (Table 6).

The mean graft survival was 13.8 years $( \pm 7.8$ years: range 6.0-21.6) from transplantation date to the last follow up, and no graft loss was observed.

\section{Discussion}

Our retrospective analysis reports clinical and histological data from a single centre cohort of kidney transplant recipients who developed $d n \mathrm{DSA}$. No improvement in histopathological parameters was observed with any therapeutic intervention. We observed a loss of kidney function and increase in cg score $\geq 1$ at follow up. Graft survival was similar in patients who received

Table 3 Overall clinical and histological changes over time $(n=22)$

\begin{tabular}{|c|c|c|c|}
\hline & $\begin{array}{l}\text { Biopsy at the } \\
\text { time of dnDSA }\end{array}$ & $\begin{array}{l}\text { Follow up } \\
\text { biopsy }\end{array}$ & $\begin{array}{l}\text { Paired } \\
t \text {-test }\end{array}$ \\
\hline $\begin{array}{l}\text { Mean eGFR using the CKD-EPI } \\
\text { equation }(\mathrm{ml} / \mathrm{min} \text {, mean }+/-\mathrm{SD})\end{array}$ & $45.9(16.7)$ & $37.4(13.8)$ & 0.005 \\
\hline$g(n, \%$ of $g \geq 1)$ & $10(45.4)$ & $11(50.0)$ & 0.47 \\
\hline $\operatorname{ptc}(n, \%$ of $p t c \geq 1)$ & $10(45.4)$ & $6(27.2)$ & 0.26 \\
\hline$g+\operatorname{ptc}(n, \%$ of $g+p t c \geq 2)$ & $10(45.4)$ & $7(31.8)$ & 0.77 \\
\hline \multicolumn{4}{|l|}{ Overall change in $\mathrm{g}+\mathrm{ptc}(\mathrm{n}, \%)$} \\
\hline o Reduction in $\mathrm{g}+$ ptc score & & $9(40.9)$ & \\
\hline - No change in $g+$ ptc score & & $7(31.8)$ & \\
\hline$\circ$ Progression in $\mathrm{g}+$ ptc score & & $6(27.3)$ & \\
\hline$t(n, \%$ of $t \geq 1)$ & $4(18.1)$ & 0 & 0.04 \\
\hline$c 4 d(n, \%$ of $c 4 d \geq 1)$ & $9(40.9)$ & $5(22.7)$ & 0.16 \\
\hline $\operatorname{cg}(n, \%$ of $c g \geq 1)$ & $6(27.2)$ & $10(45.4)$ & 0.04 \\
\hline
\end{tabular}

eGFR estimated glomerular filtration rate, CKD-EPI Chronic Kidney Disease Epidemiology Collaboration, SD standard deviation, $g$ glomerulitis, ptc peritubular capillaritis, $t$ tubulitis, $c g$ chronic glomerulopathy treatment at the time of $d n \mathrm{DSA}$ and patients who did not receive therapy.

Wiebe et al. [12] analysed the clinical and histopathological correlations with $d n \mathrm{DSA}$ in 315 consecutive renal transplants without pre-transplant DSA. The mean followup was shorter $(6.2 \pm 2.9$ years). Forty-seven of the 315 (15\%) patients developed $d n$ DSA 4.6 years post-transplant, which is earlier than our cases. They found that the two independent predictors of $d n \mathrm{DSA}$ were a mismatch for HLA-DR $\beta 1>0$ (OR 5.66, $p<0.006)$ and non-adherence (OR 8.75, $p<0.001$ ). The authors demonstrated that the median 10-year graft survival for patients with $d n$ DSA was lower than the no $d n$ DSA group $(57 \%$ vs. $96 \%, p<0.0001)$. Their study found a non-significant trend with an odds

Table 4 Overall effect of treatment on the evolution eGFR, microvascular inflammation (MVI) and chronic transplant glomerulopathy (cg); Fisher's exact test $p=0.64,0.86$ and 1.0, respectively

\begin{tabular}{|c|c|c|}
\hline & $\begin{array}{l}\text { No treatment at the } \\
\text { time of } d n D S A(n=9)\end{array}$ & $\begin{array}{l}\text { Any combination of } \\
\text { treatment at the time } \\
\text { of } d n D S A(n=13)\end{array}$ \\
\hline \multicolumn{3}{|l|}{ eGFR evolution } \\
\hline o eGFR increase & 3 & 4 \\
\hline - Stable eGFR & 1 & 0 \\
\hline o eGFR decrease & 5 & 9 \\
\hline \multicolumn{3}{|l|}{ MVI evolution } \\
\hline - MVI reduction & 3 & 6 \\
\hline o No change in MVI & 3 & 4 \\
\hline o MVI progression & 3 & 3 \\
\hline \multicolumn{3}{|l|}{ Cg evolution } \\
\hline - Cg reduction & 1 & 0 \\
\hline o No change in cg & 5 & 11 \\
\hline o Cg progression & 3 & 2 \\
\hline
\end{tabular}

eGFR estimated glomerular filtration rate, CKD-EPI Chronic Kidney Disease Epidemiology Collaboration, MVI microvascular inflammation defined as the $\mathrm{g}+\mathrm{ptc}$ score 
Table 5 Combination of treatment at the time of $\operatorname{dnDSA}(n=13)$ and overall MVI outcomes (*Fisher's exact test $p<0.05$ )

\begin{tabular}{|c|c|c|c|c|c|c|c|c|}
\hline \multirow[t]{2}{*}{$N=13$} & \multicolumn{2}{|c|}{ Rituximab } & \multicolumn{2}{|c|}{ Plasma exchanges } & \multicolumn{2}{|c|}{ IVIG } & \multicolumn{2}{|c|}{ Steroid bolus and treatment reinforcement } \\
\hline & yes & no & yes & no & yes & no & yes & no \\
\hline MVI reduction $(n=6)$ & 3 & 3 & 3 & 3 & 0 & $6^{*}$ & 5 & 1 \\
\hline No change in MVI (=4) & 1 & 3 & 1 & 3 & 3 & 1 & 2 & 2 \\
\hline MVI progression $(n=3)$ & 2 & 1 & 2 & 1 & 2 & 1 & 1 & 2 \\
\hline
\end{tabular}

IVIG intravenous immunoglobulins, $M V I$ microvascular inflammation defined as $\mathrm{g}+\mathrm{ptc}$ score

ratio of 1.57 per rejection episode prior to an occurrence of $d n$ DSA $(p=0.061)$. A high prevalence of TCMR episodes preceded the development of $d n$ DSA (36.3\%) in our study. Less than $20 \%$ of the 22 patients who developed $d n$ DSA exhibited graft dysfunction at the time of diagnosis, which is consistent with Yamamoto et al., who reported a prevalence of $40 \%$ of patients with $d n \mathrm{DSA}$ and biopsy-proven subclinical ABMR [21]. Histological features at the time of $d n$ DSA identification were not broadly consistent with ABMR in our study, and less than $50 \%$ of the biopsies exhibited an MVI score $\geq 2$. C4d was negative in $59.1 \%$ of our baseline biopsies, which is consistent with previous reports [22, 23].

Further analyses of the incidence and impact of $d n \mathrm{DSA}$ in renal transplant recipients were performed. Forty-seven of the 189 consecutive non-sensitized, non-HLA-identical patients who received a kidney transplant between March 1999 and March 2006 (25\%) developed $d n$ DSA within 10 years [24]. The cumulative incidence 5 years posttransplantation was $20 \%$, and more than half of these patients developed $d n \mathrm{DSA}$ in the first post-transplantation year. Patients of a younger age $(<35$ years old), deceased donor, the presence of HLA antibodies (non-DSA) and DQ mismatch (as previously described [25]) were most likely to develop $d n$ DSA in our study.

One strength of our analysis is the focus on the evolution of histological parameters in patients with $d n$ DSA. Focusing on cases with negative crossmatch and DSA identified 6 months post-KT avoided the analysis of patients with preformed DSA. Our data evaluated the effect of each component of treatment on the evolution of the primary features of the Banff score associated with subtypes of ABMR [26]. Halloran et al. performed a recent principal component analysis of 164 indication biopsies [26] and found 3 main ABMR phenotypes: early ABMR with MVI lesions only (called pgABMR), late
ABMR with cg lesions (cgABMR) and mixed feature (pgcgABMR).

The present study has several limitations. First, it used a retrospective analysis or different therapeutic regimens. However, we provide data on a broad heterogeneous kidney transplant recipient population that reflects routine clinical practice. Second, the small number of patients, without a control non- $d n D S A$ group, prevents us from drawing any conclusions about the best protocol to treat $d n \mathrm{DSA}$. We did not evaluate patient adherence, which is a known cause of $d n$ DSA development and eventual graft failure [4]. Finally, an obvious pitfall was the non-standardisation of our treatment following $d n \mathrm{DSA}$ and biopsy results. The current evidence is insufficient to create an internationally approved protocol for the treatment of $d n \mathrm{DSA}$. Our treatment protocol for ABMR evolved over time and reflects changes in practice based on the literature and a tailoring of therapy based on patient age, co-morbidities and cancer risk. Notably, the absence of IVIG as a component of the treatment given at the time of $d n \mathrm{DSA}$ was associated with MVI reduction. Current evidence is conflicting on the utility of IVIG in the treatment of antibody mediated injury. The benefit has been reported for active ABMR but its benefit on late/chronic ABMR is less straight forward [27].

Evidence supporting the use of rituximab for MVI or cg lesion is also scant $[27,28]$. A recent randomized double-blind, placebo-controlled trial did not demonstrate a significant advantage of rituximab in biopsyproven ABMR in creatinine level or proteinuria at 12 months [28]. The decision to treat $d n \mathrm{DSA}$ independently of histological features has not been addressed. Use of an intragraft transcript set measurement, as proposed in the 2013 Banff update [19], may further help

Table 6 Combination of treatment at the time of dnDSA $(n=13)$ and overall cg outcomes (*Fisher's exact test $p<0.05)$

\begin{tabular}{|c|c|c|c|c|c|c|c|c|}
\hline \multirow[t]{2}{*}{$N=13$} & \multicolumn{2}{|c|}{ Rituximab } & \multicolumn{2}{|c|}{ Plasma exchanges } & \multicolumn{2}{|c|}{ IVIG } & \multicolumn{2}{|c|}{ Steroid bolus and treatment reinforcement } \\
\hline & yes & no & yes & no & yes & no & yes & no \\
\hline No change in $\mathrm{cg}(n=11)$ & 5 & 6 & 4 & 7 & 4 & 7 & 6 & 5 \\
\hline Cg progression $(n=2)$ & 1 & 1 & 2 & 0 & 1 & 1 & 2 & 0 \\
\hline
\end{tabular}


the reclassification of these biopsies, but this methodology is not widely implemented yet [29].

\section{Conclusions}

We describe retrospective results from a single centre of the clinical and histological features at the time of $d n$ DSA collection with a follow up biopsy after therapeutic management. We observed no difference in histological evolution with or without treatment. Evolution was marked by a decrease in kidney function and increase in proportion of patients with transplant glomerulopathy at the follow up biopsy. No statistically significant changes in the MVI or cg parameters were observed in the follow up biopsy of patients who received different treatments. Further intervention studies are needed to prospectively evaluate the treatment stratification and integration of the $d n$ DSA status, biopsy histological and molecular features and patient's functional status and comorbidities. Novel drugs are necessary to control $d n \mathrm{DSA}$ and its long-term effect on graft function.

\section{Abbreviations}

ABMR: Antibody mediated rejection; ATG: Antithymocyte globulin; CDC: Complement-dependent cytotoxicity; dnDSA: de novo donor specific antibody; eGFR: estimated glomerular filtration rate; HLA: Human leukocyte antigen; IVIG: Intravenous immunoglobulins; MVI: microvascular inflammation; POD: post-operative day; SPA: solid phase assay; TCMR: T-cell mediated rejection

\section{Acknowledgments}

None.

\section{Funding}

No funding was received to prepare this manuscript. The authors of this manuscript have no conflicts of interest to disclose, as described by BMC Nephrology.

\section{Availability of data and materials}

The dataset generated and analysed during the current study is not publicly available because it contains information that could compromise research participant privacy and consent, but data are available from the corresponding author on reasonable request.

\section{Authors' contributions}

YB and SFL drafted the manuscript. SFL and JV performed the anti-HLA antibodies analyses. SM and OS reviewed the kidney biopsies. PYM participated in the writing of the manuscript, and $\mathrm{KH}$ managed the cases and thoughtfully revised the manuscript. All authors read and approved the final manuscript.

\section{Ethics approval and consent to participate}

The ethics committee approved the study, which was performed in accordance with the regulations of Geneva University Hospitals. The Commission cantonale d'éthique de la recherche granted approval for this retrospective study (CCER, cantonal ethical commission for research of Geneva; reference $N^{\circ}$ 6-208). Written informed consent for participation was waived because of the retrospective nature of the study.

\section{Consent for publication}

Not applicable.

\section{Competing interests}

The authors declare that they have no competing interests.

\section{Publisher's Note}

Springer Nature remains neutral with regard to jurisdictional claims in published maps and institutional affiliations.

\section{Author details}

${ }^{1}$ Division of Nephrology, Geneva University Hospitals, Rue Gabrielle Perret-Gentil 4, 1205 Geneva, Switzerland. ${ }^{2}$ Institute of Clinical Pathology, Geneva University Hospitals, Geneva, Switzerland. ${ }^{3}$ Immunology and Transplant Unit, Geneva University Hospitals, Geneva, Switzerland. ${ }^{4}$ Division of Transplantation, Geneva University Hospitals, Geneva, Switzerland.

Received: 19 September 2017 Accepted: 1 April 2018

Published online: 12 April 2018

\section{References}

1. Gondos A, Dohler B, Brenner H, Opelz G. Kidney graft survival in Europe and the United States: strikingly different long-term outcomes. Transplantation. 2013;95(2):267-74.

2. Nair R, Fraer M, Agrawal N, Suneja M. Acute transplant glomerulopathy is associated with antibody-mediated rejection and poor graft outcome. Transplant Proc. 2010;42(9):3507-12.

3. Einecke G, Sis B, Reeve J, et al. Antibody-mediated microcirculation injury is the major cause of late kidney transplant failure. Am J Transplant. 2009; 9(11):2520-31.

4. Sellares J, de Freitas DG, Mengel M, et al. Understanding the causes of kidney transplant failure: the dominant role of antibody-mediated rejection and nonadherence. Am J Transplant. 2012;12(2):388-99.

5. Riethmuller S, Ferrari-Lacraz S, Muller MK, et al. Donor-specific antibody levels and three generations of crossmatches to predict antibody-mediated rejection in kidney transplantation. Transplantation. 2010;90(2):160-7.

6. Mohan S, Palanisamy A, Tsapepas D, et al. Donor-specific antibodies adversely affect kidney allograft outcomes. J Am Soc Nephrol. 2012;23(12): 2061-71.

7. Tsapepas DS, Vasilescu R, Tanriover B, et al. Preformed donor-specific antibodies and risk of antibody-mediated rejection in repeat renal transplantation. Transplantation. 2014;97(6):642-7.

8. Aubert O, Kamar N, Vernerey D, et al. Long term outcomes of transplantation using kidneys from expanded criteria donors: prospective, population based cohort study. BMJ. 2015;351:h3557.

9. O'Leary JG, Samaniego M, Barrio MC, et al. The influence of immunosuppressive agents on the risk of De novo donor-specific HLA antibody production in solid organ transplant recipients. Transplantation. 2016;100(1):39-53.

10. Ferrari-Lacraz S, Tiercy JM, Villard J. Detection of anti-HLA antibodies by solid-phase assay in kidney transplantation: friend or foe? Tissue Antigens. 2012;79(5):315-25.

11. Almeshari K, Pall A, Chaballout A, et al. Targeted monitoring of donorspecific HLA antibodies following renal transplantation. Clin Transpl. 2011: 395-400.

12. Wiebe C, Gibson IW, Blydt-Hansen TD, et al. Evolution and clinical pathologic correlations of de novo donor-specific HLA antibody post kidney transplant. Am J Transplant. 2012;12(5):1157-67.

13. Mao Q, Terasaki PI, Cai J, et al. Extremely high association between appearance of HLA antibodies and failure of kidney grafts in a five-year longitudinal study. Am J Transplant. 2007;7(4):864-71.

14. Loupy A, Lefaucheur C, Vernerey D, et al. Complement-binding anti-HLA antibodies and kidney-allograft survival. N Engl J Med. 2013;369(13):1215-26.

15. Sicard A, Ducreux S, Rabeyrin M, et al. Detection of C3d-binding donorspecific anti-HLA antibodies at diagnosis of humoral rejection predicts renal graft loss. J Am Soc Nephrol. 2015;26(2):457-67.

16. Lefaucheur C, Suberbielle-Boissel C, Hill GS, et al. Clinical relevance of preformed HLA donor-specific antibodies in kidney transplantation. Am J Transplant. 2008;8(2):324-31.

17. Montgomery RA, Lonze BE, King KE, et al. Desensitization in HLA-incompatible kidney recipients and survival. N Engl J Med. 2011;365(4):318-26.

18. Djamali A, Kaufman DB, Ellis TM, Zhong W, Matas A, Samaniego M. Diagnosis and management of antibody-mediated rejection: current status and novel approaches. Am J Transplant. 2014;14(2):255-71.

19. Haas M, Sis B, Racusen LC, et al. Banff 2013 meeting report: inclusion of c4d-negative antibody-mediated rejection and antibody-associated arterial lesions. Am J Transplant. 2014;14(2):272-83. 
20. Colombo MB, Haworth SE, Poli F, et al. Luminex technology for anti-HLA antibody screening: evaluation of performance and of impact on laboratory routine. Cytometry B Clin Cytom. 2007;72(6):465-71.

21. Yamamoto T, Watarai Y, Takeda A, et al. De novo anti-HLA DSA characteristics and subclinical antibody-mediated kidney allograft injury. Transplantation. 2016;100(10):2194-202.

22. Halloran PF, Pereira AB, Chang J, et al. Microarray diagnosis of antibodymediated rejection in kidney transplant biopsies: an international prospective study (INTERCOM). Am J Transplant. 2013;13(11):2865-74.

23. Gupta $\mathrm{A}$, Broin $\mathrm{PO}, \mathrm{Bao} \mathrm{Y}$, et al. Clinical and molecular significance of microvascular inflammation in transplant kidney biopsies. Kidney Int. 2016;89(1):217-25.

24. Everly MJ, Rebellato LM, Haisch CE, et al. Incidence and impact of de novo donor-specific alloantibody in primary renal allografts. Transplantation. 2013;95(3):410-7.

25. Rebellato LM, Everly MJ, Haisch CE, et al. A report of the epidemiology of de novo donor-specific anti-HLA antibodies (DSA) in "low-risk" renal transplant recipients. Clin Transpl. 2011:337-40.

26. Halloran PF, Merino Lopez M, Barreto PA. Identifying subphenotypes of antibody-mediated rejection in kidney transplants. Am J Transplant. 2016;16(3):908-20.

27. Tedla FM, Roche-Recinos A, Brar A. Intravenous immunoglobulin in kidney transplantation. Curr Opin Organ Transplant. 2015;20(6):630-7.

28. Sautenet $B$, Blancho $G$, Buchler $M$, et al. One-year results of the effects of rituximab on acute antibody-mediated rejection in renal transplantation: RITUX ERAH, a multicenter double-blind randomized placebo-controlled trial. Transplantation. 2016:100(2):391-9.

29. Halloran PF, Famulski KS, Reeve J. Molecular assessment of disease states in kidney transplant biopsy samples. Nat Rev Nephrol. 2016;12(9):534-48.

\section{Ready to submit your research? Choose BMC and benefit from}

- fast, convenient online submission

- thorough peer review by experienced researchers in your field

- rapid publication on acceptance

- support for research data, including large and complex data types

- gold Open Access which fosters wider collaboration and increased citations

- maximum visibility for your research: over $100 \mathrm{M}$ website views per year 